\title{
THE
}

2016

\section{Detection of artificial water flows by the lateral line system of a benthic feeding cichlid fish}

\author{
Margot A. B. Schwalbe \\ University of Rhode Island \\ Benjamin J. Sevey \\ University of Rhode Island \\ Jacqueline F. Webb \\ University of Rhode Island, jacqueline_webb@uri.edu
}

Follow this and additional works at: https://digitalcommons.uri.edu/bio_facpubs

Terms of Use

All rights reserved under copyright.

\section{Citation/Publisher Attribution}

Schwalbe, M. A.B., Sevey, B. J., \& Webb, J. F. (2016). Detection of artificial water flows by the lateral line system of a benthic feeding cichlid fish. Journal of Experimental Viology, 219, 1050-1059: doi: 10.1242/ jeb. 136150

Available at: http://dx.doi.org/10.1242/jeb.136150

This Article is brought to you for free and open access by the Biological Sciences at DigitalCommons@URI. It has been accepted for inclusion in Biological Sciences Faculty Publications by an authorized administrator of DigitalCommons@URI.For more information, please contact digitalcommons-group@uri.edu. 


\title{
Detection of artificial water flows by the lateral line system of a benthic feeding cichlid fish
}

\author{
Margot A. B. Schwalbe ${ }^{*, \pm}$, Benjamin J. Sevey and Jacqueline F. Webb
}

\begin{abstract}
The mechanosensory lateral line system of fishes detects water motions within a few body lengths of the source. Several types of artificial stimuli have been used to probe lateral line function in the laboratory, but few studies have investigated the role of flow sensing in benthic feeding teleosts. In this study, we used artificial flows emerging from a sandy substrate to assess the contribution of flow sensing to prey detection in the peacock cichlid, Aulonocara stuartgranti, which feeds on benthic invertebrates in Lake Malawi. Using a positive reinforcement protocol, we trained fish to respond to flows lacking the visual and chemical cues generated by tethered prey in prior studies with $A$. stuartgranti. Fish successfully responded to artificial flows at all five rates presented (characterized using digital particle image velocimetry), and showed a range of flow-sensing behaviors, including an unconditioned bite response. Immediately after lateral line inactivation, fish rarely responded to flows and the loss of vital fluorescent staining of hair cells (with 4-di-2-ASP) verified lateral line inactivation. Within 2 days post-treatment, some aspects of flow-sensing behavior returned and after 7 days, flow-sensing behavior and hair cell fluorescence both returned to pre-treatment levels, which is consistent with the reported timing of hair cell regeneration in other vertebrates. The presentation of ecologically relevant water flows to assess flow-sensing behaviors and the use of a positive reinforcement protocol are methods that present new opportunities to study the role of flow sensing in the feeding ecology of benthic feeding fishes.
\end{abstract}

KEY WORDS: Flow sensing, Neuromast, Positive reinforcement, Prey detection

\section{INTRODUCTION}

The mechanosensory lateral line system is found in all fishes and in larval and adult aquatic amphibians. It detects water flows within a few body lengths and mediates a range of critical behaviors, including prey detection (reviewed in Coombs and Montgomery, 2014; Montgomery et al., 2014). The role of the lateral line system in predator-prey interactions has been established in the laboratory using hydrodynamic stimuli generated by live prey, such as the movements of free-swimming prey or the filter-feeding or respiratory currents produced by sessile or benthic invertebrate prey (e.g. Hoekstra and Janssen, 1986; Pohlmann et al., 2004; Palmer et al., 2005; Schwalbe et al., 2012; Schwalbe and Webb,

\footnotetext{
Department of Biological Sciences, University of Rhode Island, 120 Flagg Road, Kingston, RI 02881, USA

*Present address: Department of Biology, Tufts University, 200 Boston Avenue, Suite 4700, Medford, MA 02155, USA.

${ }^{\ddagger}$ Author for correspondence (margot.schwalbe@tufts.edu; margot.schwalbe@gmail.com)
}

Received 9 December 2015; Accepted 25 January 2016
2014), but these natural flows are difficult to quantify. In contrast, artificial stimuli cannot accurately replicate the complexity of stimuli generated by prey, but are controllable, repeatable and quantifiable and are thus amenable to rigorous analysis (e.g. vibrating sphere, Coombs and Janssen, 1990; Coombs et al., 2001; Nauroth and Mogdans, 2009; Mogdans and Nauroth, 2011; piston pump, McHenry et al., 2009; surface waves, Bleckmann, 1980; water jets, Janssen et al., 1990; Montgomery and Skipworth, 1997; Jordan et al., 2009). Both natural and artificial flow stimuli can produce cues for other sensory modalities (e.g. sounds or odors) that fish can detect. Therefore, the specific contribution of input to the lateral line system in prey detection behavior can be assessed by temporarily inactivating the lateral line system with chemical or pharmacological agents such as cobalt chloride or aminoglycoside antibiotics (Claas et al., 1993; Coombs et al., 2001; Mogdans and Nauroth, 2011; Schwalbe et al., 2012).

The opportunity to look at structure-function relationships in the cranial lateral line system with a focus on phenotypic distinctions in closely related species can start to shed light on adaptive trends in the evolution of the lateral line system. The cranial lateral line system of bony fishes is defined by a limited number of variations in lateral line canal morphology (reviewed in Webb, 2014). Widened lateral line canals, one of five cranial lateral line canal phenotypes, are found in a small number of benthic fishes and/or fishes that live in hydrodynamically quiet or light-limited environments (Coombs et al., 1988). Theoretical and experimental studies have demonstrated the functional differences between widened canals and the more common narrow canals (Denton and Gray, 1988, 1989; Janssen, 1997; Schwalbe and Webb, 2014), and widened canals appear to be an adaptation for prey detection (discussed in Schwalbe et al., 2012; Webb, 2014). The peacock cichlids of the genus Aulonocara have widened canals (e.g. Bird and Webb, 2014; Schwalbe and Webb, 2015). When feeding, they swim just above sandy substrates and strike directly at benthic invertebrates (not visible at the surface) and have thus been described as 'sonar feeders' (Konings, 2007). Aulonocara stuartgranti uses this same prey search strategy in the laboratory, and is able to detect live, tethered adult brine shrimp (Artemia sp.) using its lateral line system (Schwalbe et al., 2012). The movements of these prey generate both hydrodynamic and visual stimuli, which requires a consideration of the relative roles of multiple sensory systems in prey detection (Schwalbe and Webb, 2014).

Prior studies with other fish species document behavioral responses to artificial water flows presented in the water column or at the water's surface. For example, the mottled sculpin, Cottus bairdi, demonstrates an orienting response to free-swimming prey (Hoekstra and Janssen, 1985), and responds to a dipole stimulus generated by a vibrating sphere (Coombs and Janssen, 1990). The striped panchax (Aplocheilus lineatus, a killifish) detects and reorients towards the source of surface waves generated by an oscillating probe (Bleckmann et al., 1981) or a small, falling pellet 
(1 mm diameter; Schwarz et al., 2011). Similarly, blind cavefish (Astyanax mexicanus) swim toward surface disturbances generated by falling clay particles in cave pools or by a vibrating rod in the laboratory, while the sighted surface-dwelling form of this species rarely exhibits these behaviors in darkness (Yoshizawa et al., 2010).

In this study, we investigated flow-sensing behavior using artificial flows emanating from a sandy substrate. These flows provided behaviorally and ecologically relevant stimuli and were used as a proxy for benthic invertebrate prey, like those on which Aulonocara spp. normally feed in Lake Malawi (Konings, 1990, 2007). This approach allowed us to assess how the lateral line system contributes to prey detection in the absence of visual and chemical stimuli generated by live prey. Aulonocara stuartgranti were trained to identify artificial flows using a positive reinforcement protocol with a food reward. Trained fish were used to determine whether fish could detect water flows of different velocities, document variation in flow-detection behaviors, and experimentally confirm the role of the lateral line system by temporarily inactivating the system (with cobalt chloride) and then tracking the recovery of flow-sensing behavior over time.

\section{MATERIALS AND METHODS}

Adult Aulonocara stuartgranti Meyer and Riehl 1985 used in these experiments were second generation fish bred from those purchased from a commercial supplier (Bluegrass Aquatics, Louisville, KY, USA). Five fish [76-94 mm total length (TL), 2 males and 3 females] used in behavioral experiments were housed individually in large aquaria at $26 \pm 1^{\circ} \mathrm{C}$ and $1 \mathrm{ppt}$ salinity (Cichlid Lake Salt, Seachem Laboratories, Inc., Madison, GA, USA), with a 12 h:12 h light:dark cycle. Nine additional fish [25 $\mathrm{mm}$ standard length (SL), 1 juvenile, and 75-108 mm TL, all males] were used in fluorescence staining experiments (see below) and were housed in groups under the same conditions in large aquaria. All fish were fed daily with cichlid pellets (New Life Spectrum Cichlid Formula, New Life International, Inc., Homestead, FL, USA). Animal care and all experimental procedures followed an approved University of Rhode Island IACUC protocol.

\section{Generation and visualization of artificial flows}

A peristaltic pump (model 07551-00, Cole Parmer, Vernon Hills, IL, USA) generated flows, which exited from one of six holes $(1 \mathrm{~cm}$ diameter $)$ in a sheet of Plexiglas $(61 \times 46 \times 1.3 \mathrm{~cm})$ that was positioned on the bottom of a large experimental tank $(120 \times 75 \times 60 \mathrm{~cm}, 560 \mathrm{l}$; Fig. 1A). A network of chemically inert tubing $(1 \mathrm{~cm}$ internal diameter, high-density polyethylene tubing, Cole Parmer), located under the Plexiglas sheet, connected the pump to all six holes (Fig. 1B). Each of the six holes connected to the pump via tubing was paired with another hole $7 \mathrm{~cm}$ to its right, which was at the end of a short tube $(\sim 5 \mathrm{~cm}$ long, same tubing diameter as above) that was not connected to the pump, and served as a visual control. Thus, holes were arranged in six pairs (12 holes in total) and in each pair, the left hole was connected to the pump (=active hole) and the right hole was not connected to the pump (=visual control hole). Only one of the six pairs of holes was open at a time and the remaining holes were plugged with a clear silicone stopper. The relative position of the holes replicated the location of pairs of live and dead tethered prey presented to fish in previous studies (Schwalbe et al., 2012; Schwalbe and Webb, 2015).

Measures were taken to prevent fish from perceiving stimuli other than the one from which flow emerged during each trial. To address possible responses to visual cues, all holes were flush with the sand that covered the bottom of the experimental tank and the Plexiglas sheet. To limit responses to olfactory cues, tank water was placed in a bucket that was the source of water circulated through the tubing during experiments. To eliminate responses to thermal cues, the water supply in the bucket was changed frequently as room temperature was a few degrees cooler than the experimental tank. Finally, in order to reduce mechanical noise, the peristaltic pump was on a separate table adjacent to the tank (Fig. 1).

Normal peristaltic pump operation created pulsed flows, with fluctuations generated by the compression of the tubing against rollers with rotations of the pump head. Flow rates were calculated as $V=\dot{Q} / A$, where $V$ is water velocity, $\dot{Q}$ is flow rate (controlled by the digital settings of the pump, in $\mathrm{ml} \mathrm{min}^{-1}$ ) and $A$ is the internal cross-sectional area of the tubing $\left(31.67 \mathrm{~mm}^{2}\right)$. The pump flow rates selected based on this equation were 1.9, 4.8, 9.5, 19.0 and $38.0 \mathrm{ml} \mathrm{min}{ }^{-1}$, which correspond to velocities of 1, 2.5, 5, 10 and $20 \mathrm{~mm} \mathrm{~s}^{-1}$, respectively. This range of velocities includes those generated by different free-swimming and tethered invertebrates, such as foraging copepods $\left(2-6 \mathrm{~mm} \mathrm{~s}^{-1}\right.$; van Duren and Videler, 2003), tethered krill ( $\sim 50 \mathrm{~mm} \mathrm{~s}^{-1}$; Yen et al., 2003), swimming copepodids ( $\sim 12 \mathrm{~mm} \mathrm{~s}^{-1}$; Catton et al., 2007), midge larvae in a

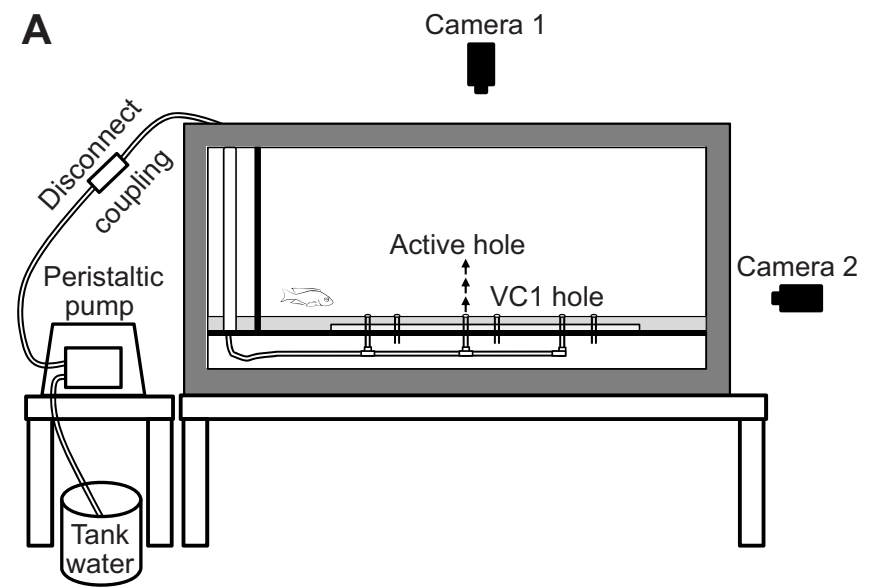

B

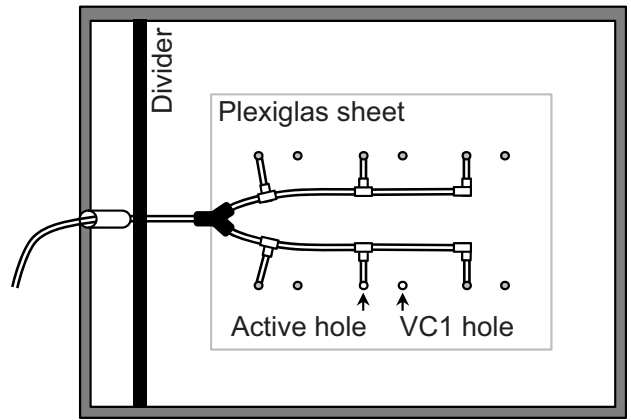

Fig. 1. Apparatus designed for this study. (A) Configuration of the apparatus in the experimental tank for a trial with flow, showing the peristaltic pump on an adjacent table. Six pieces of tubing are attached to a supply of tank water and the peristaltic pump, and six other holes are not attached to the water supply and can serve as visual controls (VC1, VC2). For trials with diverted flow, the tubing is disconnected at the 'disconnect coupling' and no flow from the peristaltic pump enters the experimental tank. Camera 1 was positioned directly over the tank, while camera 2 was placed laterally at the level of the sand and in front of the tank window. (B) Dorsal view of the apparatus showing the configuration of the tubing and placement of the pairs of holes. One pair of holes is open (arrows; active and VC1) while all other holes are plugged with silicone stoppers. The tubing and Plexiglas sheet, illustrated here, were covered with sand and were not visible to the fish. 
burrow ( $\sim 14 \mathrm{~mm} \mathrm{~s}^{-1}$; Morad et al., 2010) and tethered adult brine shrimp (2-7 $\mathrm{mm} \mathrm{s}^{-1}$; Schwalbe et al., 2012).

Digital particle image velocimetry (DPIV) was used to analyze the water flowing from the active holes. The Plexiglas sheet holding the network of tubing was placed in a 1501 glass tank and water was seeded with near-neutrally buoyant, silver-coated reflective particles (12-14 $\mu \mathrm{m}$ diameter; Potters Industries, Inc., Parsipanny, NJ, USA) and at a density of $0.1 \mathrm{~g} \mathrm{l}^{-1}$ in the tank. For each hole, a light beam from a continuous $1000 \mathrm{~mW}$ krypton-ion laser (Spyder III Krypton, Wicked Lasers, Kowloon, Hong Kong) was focused into a $2 \mathrm{~mm}$ thick and $10 \mathrm{~cm}$ wide vertical sheet that illuminated the diameter of the hole. A high-speed, high-resolution $(1024 \times 512$ pixels) Photron APX camera (Photron USA, San Diego, CA, USA) was positioned perpendicular to the laser sheet to record particle movement at 60 frames $^{-1}$ while the peristaltic pump was running for at least $8 \mathrm{~s}$. Images were processed with DaVis software (v8, LaVision, Goettingen, Germany) using sequential cross-correlation without pre-processing. An initial correlation window of $32 \times 32$ pixels was selected with multi-pass with decreasing size to a final interrogation window of $16 \times 16$ pixels with $50 \%$ overlap. The mean and maximum flow velocities were measured within a small area $(1 \times 0.5 \mathrm{~cm})$ directly above each active tube $(0.5 \mathrm{~cm})$ at five flow rates (1.9-38.0 ml min $\left.{ }^{-1}\right)$. Mean and maximum shear rate were measured in the same small area. This was calculated from the partial derivative $\partial v / \partial x$ based on velocity measurements:

$$
\frac{\partial v}{\partial x} \approx \frac{V}{(1 / 2) W},
$$

where $V$ is water velocity (mean or maximum) and $W$ is the width of the water jet exiting the active tube. The area analyzed represents the location where A. stuartgranti positioned their lower jaws over an open tube with flow generated by the pump and also tethered prey as in prior studies (Schwalbe et al., 2012).

\section{Training protocol}

Aulonocara stuartgranti $(N=5)$ were trained to respond to a flow using a four-step positive reinforcement protocol. A fish did not progress to the next step in the training protocol until it could successfully repeat a task $\geq 70 \%$ of the time. In step 1 , in its home tank, a fish was presented with a feeding wand (R2 Target Feeder Wand, R2 Fish School, Orlando, FL, USA) and received food (cichlids pellets, same as above) from a small slot in its tip. The fish received a reward (1 pellet) for swimming directly towards the wand upon its insertion into the tank and for following the wand for several seconds. In step 2, the fish was transferred to the experimental tank, and was rewarded with food (one pellet) after heading directly towards the wand and following the wand. In step 3 , the fish was transferred to the experimental tank, and was rewarded ( 1 pellet) after following the wand and holding position over a tube with flow $\left(28.5 \mathrm{ml} \mathrm{min}^{-1}\right)$ for $\geq 3 \mathrm{~s}$. In step 4 , the fish was transferred to the experimental tank, and was allowed to search for a tube with flow for $15 \mathrm{~min}$. If the fish positioned its lower jaw over the correct tube for $\geq 3 \mathrm{~s}$, the feeding wand was immediately inserted into the tank ( $>30 \mathrm{~cm}$ away from the tube with flow) and the fish immediately received a reward (1 pellet). This provided enough time for the flows to dissipate after the pump was turned off, and to reload the feeding wand for the next training bout $(\sim 1 \mathrm{~min})$. High motivation levels were maintained by feeding fish no more than 12 pellets per day. In both steps 3 and 4, the position of the water flow was randomly selected from among the six tubes connected to the pump (the others were plugged) and was changed after every three rewards or after $15 \mathrm{~min}$ to avoid positional bias. Each fish was subjected to daily training ( 5 times per week), which progressed from step 1 to step 4 over a period of $4-6$ weeks. A fish was successfully trained when it demonstrated $\geq 70 \%$ correct responses to flows in step 4 and training continued 2-3 times per week in the experimental tank to maintain the step 4 conditioned behavior.

\section{Experimental design}

A behavioral session was defined as a sequence of 12 trials. In nine of the 12 trials within a session, flow emerged from an open hole connected to the peristaltic pump (=active hole) and no flow emerged from the control hole $7 \mathrm{~cm}$ to its right (=visual control 1, $\mathrm{VC} 1$ ). In the other three trials in a session, flow was diverted to a bucket before it could reach the open hole (=inactive hole), thus serving as a control for positional bias and mechanical noise; the hole $7 \mathrm{~cm}$ to its right was open, but was not connected to the pump (=visual control 2, VC2). After every three trials, the two holes in the pair were plugged and a different pair of holes was unplugged to avoid positional bias. Thus, the location of the open pair of holes and whether or not flow emerged from the left hole of the pair was the same for three consecutive trials, after which a different pair of holes (and the order of trials with flow or diverted flow) was randomly chosen.

At the start of a trial, one randomly selected fish was moved into the experimental tank and acclimated for $\sim 5 \mathrm{~min}$. Once the fish began to explore the tank, the pump was turned on to start the flow of water. A trial with flow ended after a fish responded to the flow emerging from the active hole by remaining over it for $\geq 3 \mathrm{~s}$ and a food reward was given, or after $3 \mathrm{~min}$ if the fish did not respond to the active hole. A trial with diverted flow ended after $3 \mathrm{~min}$. At the end of each trial, the pump was turned off to allow flows to dissipate. At the end of the final (twelfth) trial in a session, the fish was returned to its home tank and the next fish was moved into the experimental tank for a new session.

Behavior was recorded with two high-definition video cameras (Sony, HDR-CX550V, 30 frames $^{-1}$ ) positioned to provide a dorsal or a lateral view of the experimental arena. Behavioral responses were defined and scored based on video in dorsal and lateral views. Video was analyzed using Adobe Premier Pro (CS5, Adobe Systems, San Jose, CA, USA). A light bulb wired to a manual switch was within the field of view of the dorsally positioned camera and out of view of the fish to indicate the start and end of a trial.

\section{Experiment I: effect of flow rate on flow-sensing behavior}

Sessions were carried out using four fish (TL=76-94 mm, 2 males, 2 females) and sequential sessions used decreasing flow rates (38.0, 19.0, 9.5, 4.8 and $\left.1.9 \mathrm{ml} \mathrm{min}^{-1}\right)$. Only one flow rate was presented per session, one session was done per day, and the same flow rate was used for all four fish on a given day. This flow rate sequence was then replicated such that all testing was carried out over a period of 14 days. With two possible responses per trial (to the two holes in a pair), 12 trials per session, one session for each of five flow rates, and two replicates of the entire flow rate sequence, there were 240 possible responses per fish. Four different fish were used, so the dataset included 960 potential behavioral responses.

\section{Experiment Il: effect of lateral line inactivation on flow- sensing behavior}

Five fish (the four fish used in experiment I, plus one additional trained female, $89 \mathrm{~mm} \mathrm{TL}$ ) were used in daily sessions (composed of 12 trials, as in experiment I) before and after lateral line 
inactivation using cobalt chloride. All sessions used a flow rate of $4.8 \mathrm{ml} \mathrm{min}-1$, which was within the range of flows produced by tethered live prey in a previous study (Schwalbe et al., 2012). First, each fish was run through a session on each of the 2 days before cobalt chloride treatment (days -2 and -1 ) to establish a behavioral baseline. Then, each fish was treated with $0.1 \mathrm{mmol}^{-1}$ cobalt (II) chloride heptahydrate $\left(\mathrm{CoCl}_{2}\right.$; Sigma-Aldrich, St Louis, MO, USA) for $3 \mathrm{~h}$ in a bucket of conditioned tap water and then run through a session within $2 \mathrm{~h}$ (day 0). Each fish was then run through a session each day post-treatment for 11 days (days 1-11) and then on days 14,18 and 21 to document the recovery of flow-sensing behavior. With responses to the two holes in a pair, 12 trials per session, one trial per day with each of five fish, and 17 days of sessions, the dataset analyzed included 2040 total possible behavioral responses. This experiment was carried out more than 3 months after experiment I, so fish needed to be trained 2-3 times per week in order to maintain their responses to artificial flows prior to this experiment.

Several additional adult $A$. stuartgranti $(N=7,75-108 \mathrm{~mm}$ TL, all males) were treated with $\mathrm{CoCl}_{2}$ (as above) and stained with 4-Di-2-ASP (4-[4-diethylaminostyryl]-1-methylpyridinium iodide, Sigma-Aldrich; a vital fluorescent mitochondrial stain) on different days post-treatment to confirm the effects of $\mathrm{CoCl}_{2}$ treatment on the sensory hair cells of the neuromasts. Fish were stained with $63 \mu \mathrm{mol} 1^{-1} 4$-Di-2-ASP in tank water for $5 \mathrm{~min}$ either on day 0 (within $2 \mathrm{~h}$ of treatment) or on day 3, 6, 7 or 9 post-treatment ( $N=7$ fish). Two additional fish (114 mm TL, 1 male, and $25 \mathrm{~mm} \mathrm{SL}, 1$ juvenile) were placed in tank water without $\mathrm{CoCl}_{2}$ for $3 \mathrm{~h}$ and stained with 4-Di-2-ASP (as above) and DASPEI (2-[4-(dimethylamino)styryl]-1-ethylpyridinium iodide, Sigma-Aldrich; $0.01 \%$ for $30 \mathrm{~min}$ ), respectively, as a control. Each fish was euthanized with an overdose of buffered MS-222 (ethyl 3-aminobenzoate methanesulfonate salt, Sigma-Aldrich) after fluorescence staining. Canal (revealed by dissection) and superficial neuromasts on the lateral and ventral surfaces of the head were imaged using a Nikon dissecting microscope (model SMZ 1500) equipped with an epifluorescence light source (GFPB, Ex 470/40) and a Spot camera (model 25.2 Mp Color Mosaic, Spot software v5.0). Images of neuromasts from different fish revealed the relative proportion of fluorescing (live) hair cells in neuromasts after different times following treatment with $\mathrm{CoCl}_{2}$.

\section{Statistical analysis}

Generalized linear mixed models (GLMMs, SPSS, v22, IBM, Armonk, NY, USA) were used to analyze behavioral responses to each of the four hole types (active hole and $\mathrm{VC} 1$ hole, in trials with flow; inactive hole and VC2 hole, in trials with diverted flow) in both experiments. GLMMs were performed to determine whether there was variation in the number of responses among hole types and among the different flow rates in experiment $\mathrm{I}$, and among all trial days before and after $\mathrm{CoCl}_{2}$ treatment (day -2 to day 21) in experiment II. The data were categorical, so analyses designed for normally distributed data could not be carried out. Thus, GLMM analyses were completed with either a binomial distribution (with data points for either a 'response' or a 'non-response') or a multinomial distribution (with four different types of responses: non-response, search, shift or hover; see Results for a description of behaviors) using probit link functions and first-order autoregressive covariance structure. These GLMMs were selected because they generated the lowest corrected Akaike information criterion (AICc). Further, these models predicted the probabilities of the possible outcomes for a categorically distributed dependent variable (e.g. number of responses or type of responses), given a set of independent variables (hole type and flow rate in experiment I, hole type and day in experiment II) and accounted for repeated measures from the same individual. Pairwise post hoc comparisons (using least significant differences, LSD) were carried out after examining the results from the GLMMs in experiment II to detect differences in the number of 'responses' and 'non-responses' among trial days before and after $\mathrm{CoCl}_{2}$ treatment. Differences were considered to be significant at $P<0.05$ for all statistical tests. Values are given as means \pm s.e.m., unless otherwise specified.

\section{RESULTS}

\section{DPIV quantification of artificial flows}

The peristaltic pump generated flows that were visualized and quantified using DPIV (Fig. 2A,B). The five flow rates selected

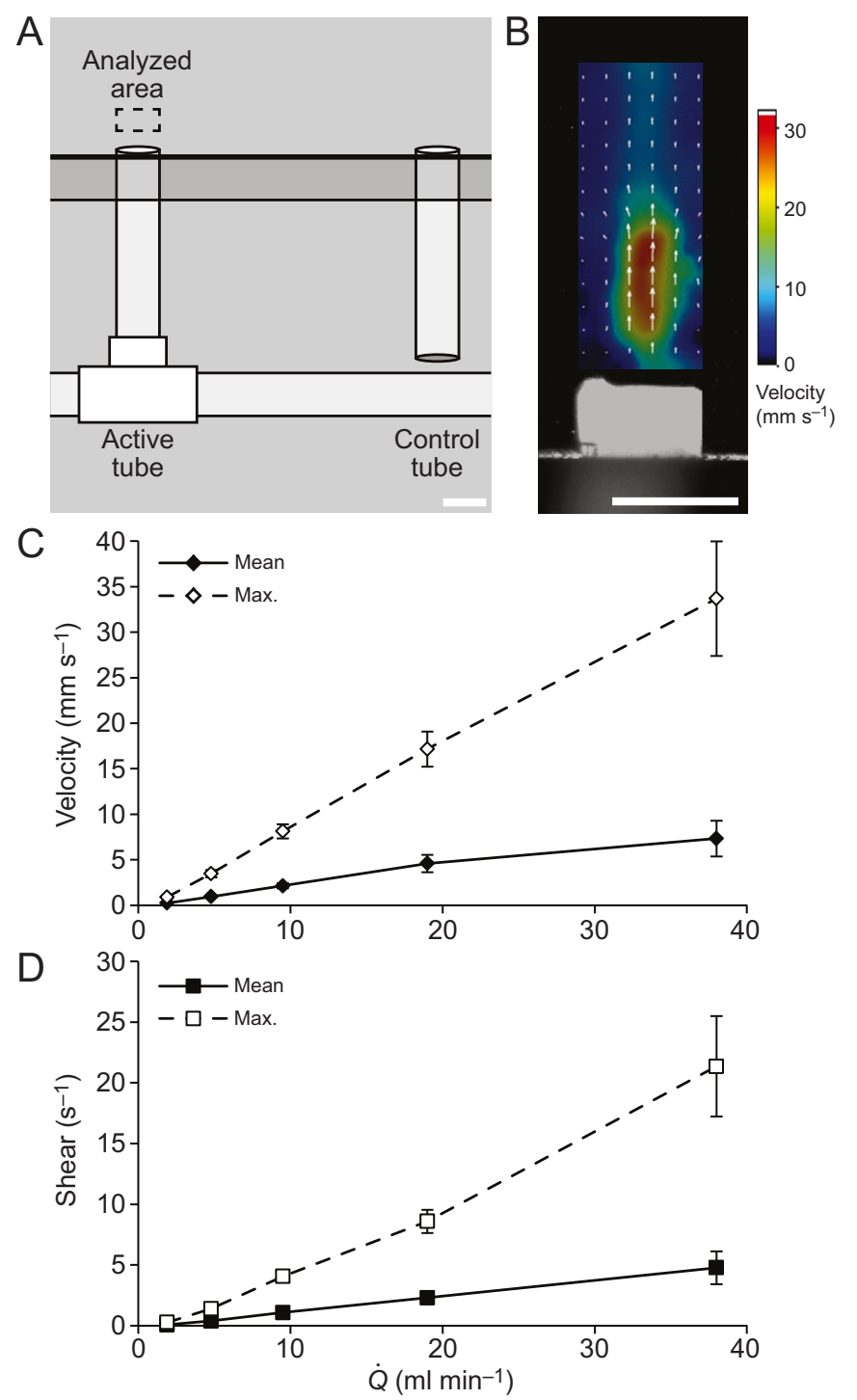

Fig. 2. Visualization of flow from the apparatus using digital particle image velocimetry (DPIV). (A) Flows were analyzed as they emerged vertically from an active hole $(1 \times 0.5 \mathrm{~cm}$ area above the hole), as indicated by the dashed box. (B) Velocity vector fields (arrows) and maps of flow magnitudes (color scale) above an active hole (red=maximum, $30 \mathrm{~mm} \mathrm{~s}^{-1}$; dark blue=minimum) at a flow rate $(\dot{Q})$ of $19.0 \mathrm{ml} \mathrm{min}^{-1}$. (C,D) Mean and maximum velocity $(C)$ and shear rate $(D)$ measured by DPIV analysis for flow rates used in the current study. Symbols in C and D represent means ss.e.m. 
Table 1. Generalized linear mixed model results for experiments I and II

\begin{tabular}{|c|c|c|c|c|c|c|c|c|c|}
\hline & \multicolumn{3}{|c|}{ Number of responses } & \multicolumn{3}{|c|}{ Types of responses } & \multicolumn{3}{|c|}{ Number of bites } \\
\hline & $F$ & d.f. & $P$ & $F$ & d.f. & $P$ & $F$ & d.f. & $P$ \\
\hline \multicolumn{10}{|l|}{ Experiment I } \\
\hline Flow rate & 0.112 & $4,1,916$ & 0.978 & 2.724 & 4,912 & 0.028 & 2.607 & 4,916 & 0.034 \\
\hline Flow ratexhole type & 1.005 & $12,1,916$ & 0.442 & 1.980 & 12,912 & 0.023 & 1.890 & 12,916 & 0.032 \\
\hline \multicolumn{10}{|l|}{ Experiment II } \\
\hline Hole type & 257.746 & $3,1,852$ & $<0.001$ & 356.096 & $3,1,848$ & $<0.001$ & 179.015 & $3,1,852$ & $<0.001$ \\
\hline
\end{tabular}

Experiment I: effect of flow rate on flow-sensing behavior. Experiment II: effect of lateral line inactivation on flow-sensing behavior.

Data are for the number of behavioral responses, types of responses and number of bites by Aulonocara stuartgranti to different hole types (active, VC1, inactive and VC2 holes) among flow rates $\left(1.9-38.0 \mathrm{ml} \mathrm{min}^{-1}\right)$ in experiment I ( $N=4$ fish) and among days before (days $\left.-2,-1\right)$ and after (days $\left.0-11,14,18,21\right)$ treatment with $\mathrm{CoCl}_{2}$ in experiment II ( $N=5$ fish).

Significant results $(P<0.05)$ are in bold.

for this study had mean and maximum flow rates over time ranging from $0.3 \pm 0.04$ and $1.0 \pm 0.07 \mathrm{~mm} \mathrm{~s}^{-1}$ at a rate of $1.9 \mathrm{ml} \mathrm{min}^{-1}$, to $7.3 \pm 2.0$ and $33.7 \pm 6.3 \mathrm{~mm} \mathrm{~s}^{-1}$ at a rate of $38.0 \mathrm{ml} \mathrm{min}{ }^{-1}$, respectively (Fig. 2C). Estimated flow velocities (calculated by $V=\dot{Q} / A$ ) did not match the mean or maximum flow rates calculated using DPIV for any of the five flow rates tested, indicating that the flow was not uniform over time and that the peristaltic pump generated pulsed flows. The average and maximum flow velocity and shear rate emerging from the active hole varied proportionally with flow rate (Fig. 2C,D). As flow
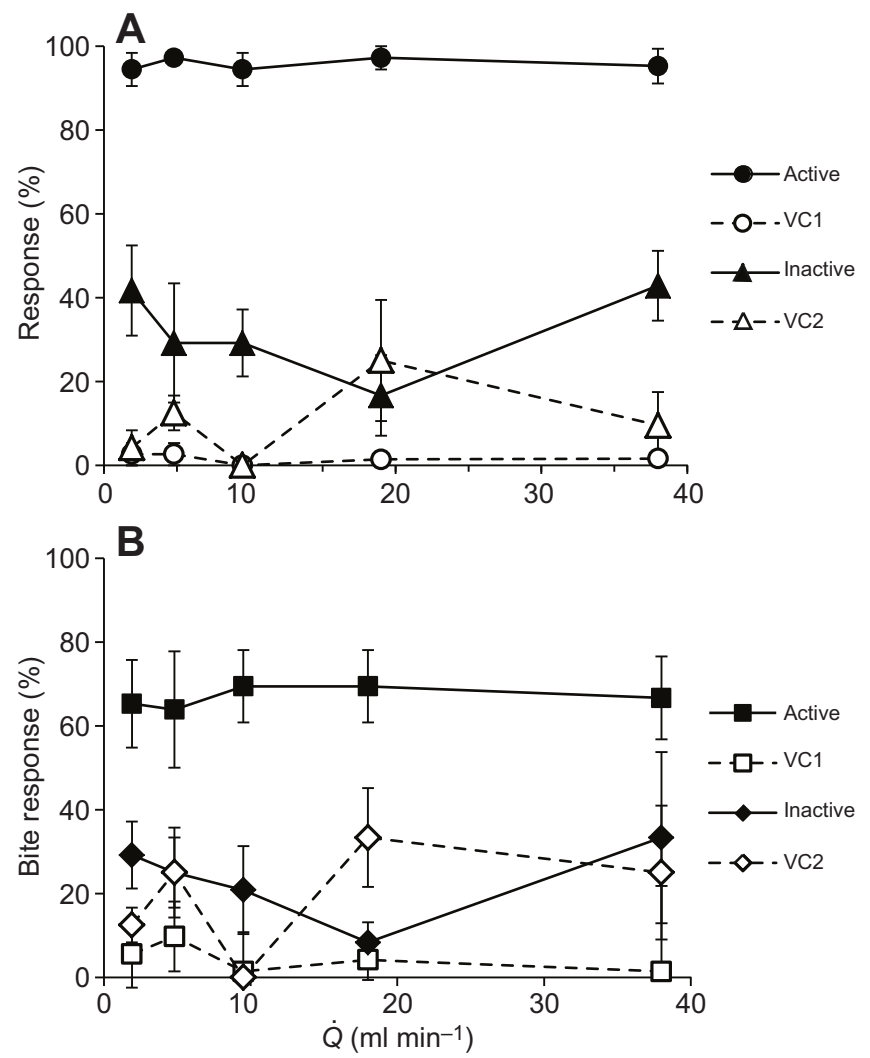

Fig. 3. Experiment $\mathrm{I}$ - percentage of responses as a function of flow rate. Percentage of all responses $(\mathrm{A})$ and percentage of unconditioned bite responses (B) by Aulonocara stuartgranti ( $N=4$ fish) to active and VC1 holes (trials with flow), and inactive and VC2 holes (trials with diverted flow) for different flow rates $(Q)$. Symbols represent the mean ( \pm s.e.m.) response (A) and unconditioned bite response (B) based on each fish's mean response for each hole type and flow rate. rate was dependent on the rate of rotation of the peristaltic pump, the pulse frequency was slower at lower flow rates than at higher flow rates. The pulse frequency was $0.04 \mathrm{~Hz}$ at a rate of $1.9 \mathrm{ml} \mathrm{min}{ }^{-1}$, compared with a higher frequency of $0.65 \mathrm{~Hz}$ at a higher rate of $38.0 \mathrm{ml} \mathrm{min}{ }^{-1}$.

\section{Definition of behavioral responses to flows}

Fish actively explored the experimental tank and frequently approached the majority of the 12 holes (six pairs of holes) during all trials in all sessions. Fish demonstrated a range of behaviors when reacting to the active hole and $\mathrm{VC} 1$ hole in trials with flow and to the inactive hole and VC2 hole in trials with diverted flow. These reactions were defined as either "nonresponses' (fish showed no movement towards the hole or stayed over the hole for $<3 \mathrm{~s}$ ) or 'responses' (fish stayed over the hole for $\geq 3 \mathrm{~s}$ ). 'Responses' were, in turn, categorized as one of three behaviors (search, shift or hover) and further characterized as to whether or not the fish bit at the tubing (an unconditioned response). A 'search' response was defined as a fish briefly pausing with its lower jaw just above a hole, then swimming away ( $>1$ body length), and quickly returning to the same hole. A 'shift' response was defined as a fish moving its lower jaw slowly from side to side directly above a hole. A 'hover' response was defined as a fish remaining stationary with its lower jaw directly over a hole.

\section{Experiment I: effect of flow rate on flow-sensing behavior}

Fish responded to flows at all flow rates presented, but the number (Fig. 3A) and type (Fig. 4) of responses varied among reactions to the four hole types: active and VC1 holes in trials with flow and inactive and VC2 holes in trials with diverted flow $(P<0.001$, GLMM; Table 1). Overall, fish responded more often to the active hole $(>94 \%)$ than to its visually similar VC1 hole $(<5 \%)$ in trials with flow. Further, in trials with diverted flow, responses to the inactive hole and its visually similar VC2 hole were much less frequent than responses to the active hole in trials with flow at the same flow rate ( $<43 \%$ for inactive hole, $<42 \%$ for $\mathrm{VC} 2$ hole). Flow rate appeared to have little influence on the number of responses to all hole types ( $P>0.05$, GLMM; Table 1, Fig. 3A), but the types of responses that fish demonstrated at the four hole types varied among flow rates ( $P=0.023$, GLMM; Table 1, Fig. 4). The majority of responses to the active hole (in trials with flow) were hover responses, which was in contrast to the responses to the other three hole types $(P<0.001$, GLMM; Table 1). The infrequent responses to the VC1 hole (in trials with flow) and to the inactive and VC2 holes (in trials with diverted flow) consisted of a combination of behaviors (Fig. 4). The bite response was unexpected; fish bit more frequently at the active hole 


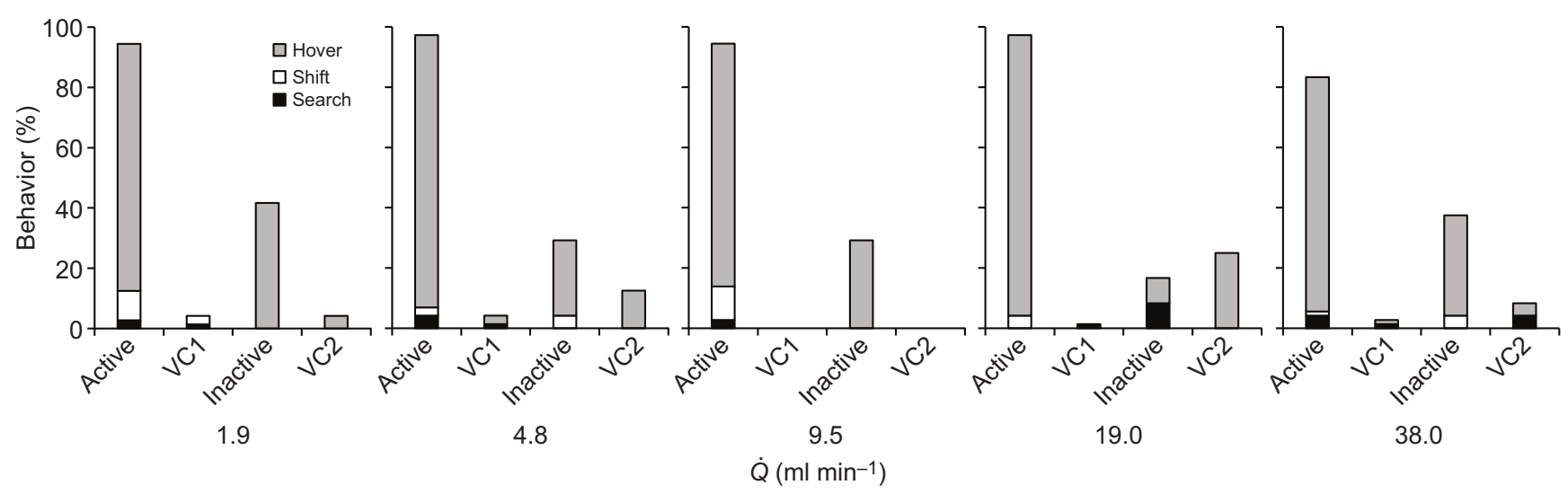

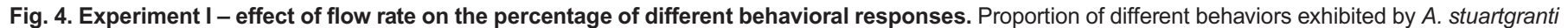

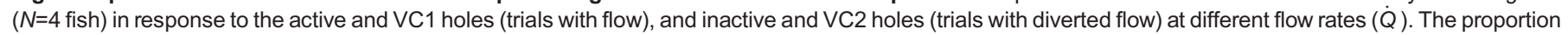
of total responses is based on the mean of each fish's behavioral response for each hole type and flow rate.

than at the other three hole types (64-69\% and $<33 \%$, respectively) at all flow rates $(P<0.001$, GLMM; Table 1, Fig. 3B).

\section{Experiment Il: effect of lateral line inactivation on flow- sensing behavior}

On the 2 days before cobalt chloride $\left(\mathrm{CoCl}_{2}\right)$ treatment (days $\left.-2,-1\right)$, fish actively explored the experimental tank and investigated the four hole types in trials with flow and in trials with diverted flow. Further, the number and type of responses varied significantly among the four hole types in each session $(P<0.001$, GLMM; Table 1$)$, as in experiment I. Following $\mathrm{CoCl}_{2}$ treatment, the number (Fig. 5A) and type (Fig. 6) of responses to the four hole types changed dramatically $\left(P<0.001\right.$, GLMM; Table 1). Immediately following $\mathrm{CoCl}_{2}$ treatment (day 0 ), the number of responses to the active hole was significantly reduced and did not return to pretreatment levels until day 3 (LSD, $P<0.05$; Table 2, Fig. 5A). Further, for several days following treatment, fish exhibited a different proportion of responses to the four hole types $(P<0.001$, GLMM; Table 1, Fig. 6). In addition, fish performed more search and shift responses than hover responses when responding to the active hole on days $0-6$, but after 1 week (days 7-21), behavior changed such that fish most commonly responded to the active hole with a hover response (Fig. 6). In addition, the number of bites at the active hole was significantly reduced following $\mathrm{CoCl}_{2}$ treatment $(P<0.001$, GLMM; Table 1), but this unconditioned behavior returned in 2 days (Fig. 5B). At day 7, the combination of a high number of hover responses and bites at the active hole indicated a return to pre-treatment behavioral responses.

Before $\mathrm{CoCl}_{2}$ treatment, fluorescence staining of neuromasts with 4-Di-2-ASP revealed the presence of live hair cells in the center of both canal and superficial neuromasts (Fig. 7A,B). Immediately following $\mathrm{CoCl}_{2}$ treatment, fluorescence was dramatically reduced (day 0 ; Fig. 7C), but superficial neuromasts fluoresced with the same intensity as those in control fish on day 3 (data not shown). By days 3 and 6 , fluorescence of canal neuromasts had partially returned, but obvious
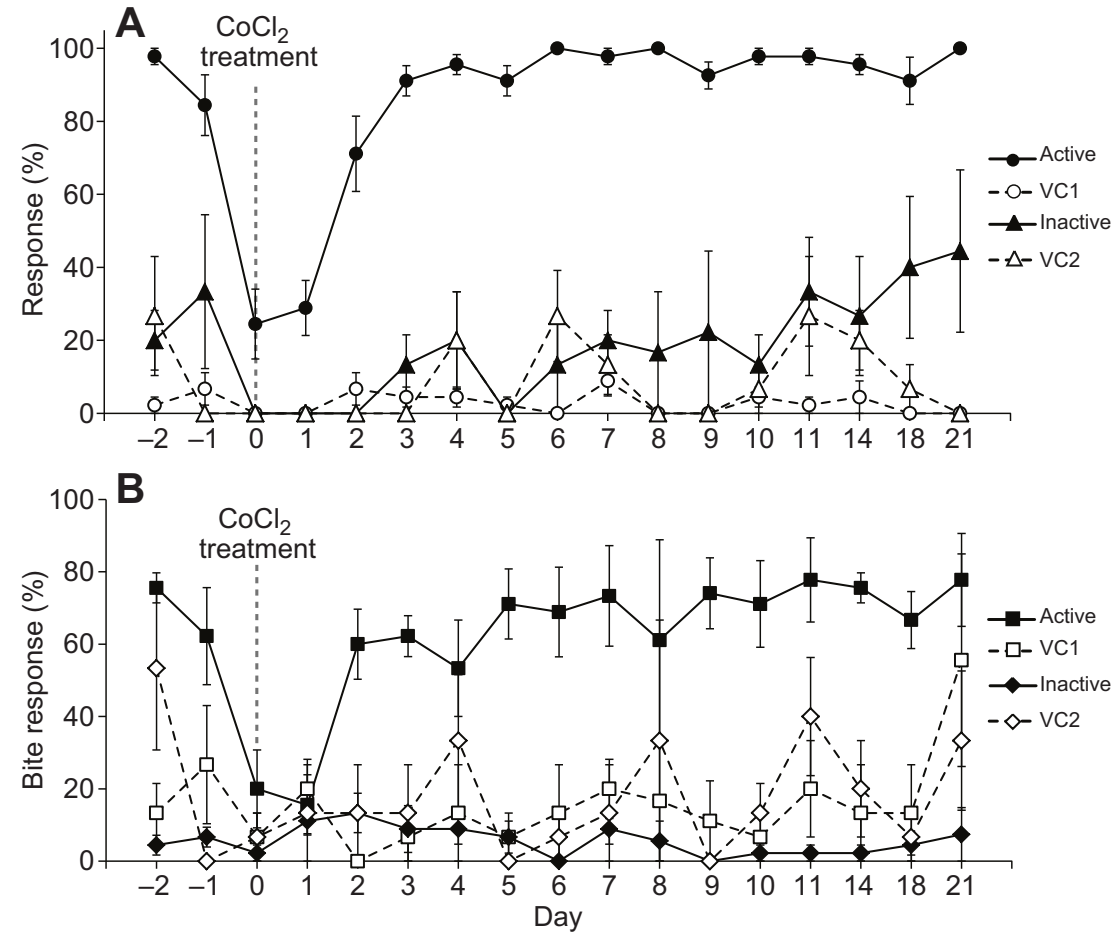

Fig. 5. Experiment II - effect of lateral line inactivation on the percentage of responses to flow over time. Percentage of all responses $(A)$ and percentage of unconditioned bite responses only (B) by $A$. stuartgranti ( $N=5$ fish) to active and VC1 holes (trials with flow), and inactive and VC2 holes (trials with diverted flow) for each day before and after treatment with cobalt chloride $\left(\mathrm{CoCl}_{2}\right)$. A flow rate of $4.8 \mathrm{ml} \mathrm{min}^{-1}$ was used in all sessions and each fish completed one session per day. Day 0 sessions were carried out within $2 \mathrm{~h}$ of $\mathrm{CoCl}_{2}$ treatment as indicated by the vertical dashed line. Symbols represent the mean ( \pm s.e.m.) response $(A)$ and unconditioned bite response (B) based on each fish's mean response for each hole type on each day. 
Table 2. Matrix of post hoc pairwise comparisons (LSD) results for the number of responses to active holes by $A$. stuartgranti ( $N=5$ fish) on days -2 to 21

\begin{tabular}{|c|c|c|c|c|c|c|c|c|c|c|c|c|c|c|c|c|c|c|}
\hline & & \multicolumn{17}{|c|}{ Day } \\
\hline & & -2 & -1 & 0 & 1 & 2 & 3 & 4 & 5 & 6 & 7 & 8 & 9 & 10 & 11 & 14 & 18 & 21 \\
\hline \multirow[t]{17}{*}{ Day } & -2 & & ns & * & * & * & ns & ns & * & ns & ns & ns & ns & ns & ns & ns & $\mathrm{ns}$ & $\overline{\mathrm{ns}}$ \\
\hline & -1 & & & * & * & * & ns & ns & ns & ns & ns & ns & ns & ns & * & ns & ns & ns \\
\hline & 0 & & & & ns & * & * & * & * & * & * & * & * & * & * & * & * & * \\
\hline & 1 & & & & & * & * & * & * & * & * & * & * & * & * & * & * & * \\
\hline & 2 & & & & & & * & * & * & * & * & ns & ns & * & * & * & * & * \\
\hline & 3 & & & & & & & ns & ns & ns & * & ns & ns & ns & * & ns & ns & * \\
\hline & 4 & & & & & & & & * & ns & * & ns & ns & ns & ns & ns & ns & ns \\
\hline & 5 & & & & & & & & & * & ns & ns & ns & ns & * & * & ns & ns \\
\hline & 6 & & & & & & & & & & ns & ns & ns & ns & ns & ns & ns & ns \\
\hline & 7 & & & & & & & & & & & ns & ns & ns & ns & ns & ns & ns \\
\hline & 8 & & & & & & & & & & & & ns & ns & ns & ns & ns & ns \\
\hline & 9 & & & & & & & & & & & & & ns & ns & ns & ns & ns \\
\hline & 10 & & & & & & & & & & & & & & ns & ns & ns & $\mathrm{ns}$ \\
\hline & 11 & & & & & & & & & & & & & & & ns & ns & ns \\
\hline & 14 & & & & & & & & & & & & & & & & ns & ns \\
\hline & 18 & & & & & & & & & & & & & & & & & ns \\
\hline & 21 & & & & & & & & & & & & & & & & & \\
\hline
\end{tabular}

${ }^{*} P<0.05 . \mathrm{ns}$, not significant.

gaps were still present between hair cells (Fig. 7D,E). On days 7 and 9, fluorescence of canal neuromasts had returned to intensity levels similar to those in control fish (Fig. 7F,G).

\section{DISCUSSION}

This study has demonstrated that the peacock cichlid, A. stuartgranti can be successfully trained to identify artificial water flows at a range of velocities and responds to such flows with several definable behaviors, which require a functional lateral line system. The role of the lateral line system in these behaviors was confirmed by inactivating the lateral line system with cobalt chloride, which eliminates flow-sensing behavior. The behavior then recovered over a time period that was consistent with the process of hair cell regeneration observed in other vertebrates

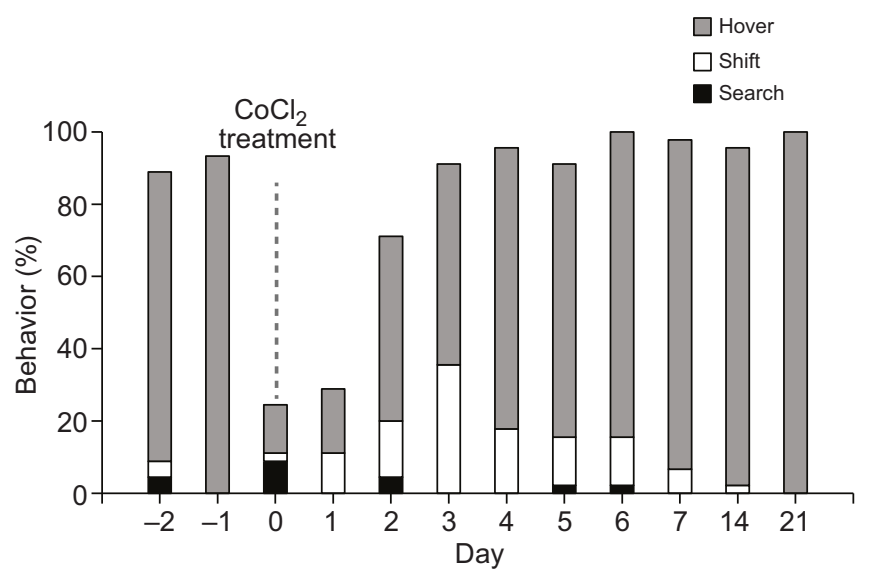

Fig. 6. Experiment II - effect of lateral line inactivation on the percentage of different behavioral responses to flow over time. Proportion of different behaviors exhibited by $A$. stuartgranti ( $N=5$ fish) in response to the active hole in trials of sessions carried out before and after treatment with $\mathrm{CoCl}_{2}$. Day 0 trials were carried out within $2 \mathrm{~h}$ of $\mathrm{CoCl}_{2}$ treatment, as indicated by the vertical dashed line. Results were similar among days 7-21, so only a subset of data from these days is included (see also Fig. 5 and Tables 1 and 2). The proportion of total responses is based on the mean of each fish's behavioral response for the active hole by day. (reviewed in Brignull et al., 2009; Lush and Piotrowski, 2014; Atkinson et al., 2015).

In the field, peacock cichlids (Aulonocara spp.) detect and strike at invertebrate prey while swimming just above sandy substrates (Konings, 2007); in the laboratory, they also swim just above a sandy substrate, and $A$. stuartgranti detect the flows generated by live tethered brine shrimp (Schwalbe et al., 2012; Schwalbe and Webb, 2015). In the current study, A. stuartgranti showed the same swimming behavior, and was successfully trained to detect water flows emerging from the sandy substrate. Aulonocara spp. likely detect flows with the canal neuromasts in the ventrally directed mandibular canal (Schwalbe et al., 2012), as well as the lower preopercular and infraorbital canals, and perhaps with the clusters of very small superficial neuromasts located in the epithelium over the mandibular lateral line canal. However, the role of different types (canal versus superficial) and groups (e.g. different series of superficial neuromasts) of neuromasts is not currently known, and can only be established with selective inactivation in future experiments.

Aulonocara stuartgranti did not show unconditioned responses to artificial flows, but after training, was able to detect artificial water flows at response rates comparable to both conditioned and unconditioned responses in other species elicited using various types of artificial hydrodynamic stimuli (mottled sculpin, Coombs et al., 2001; rays, Jordan et al., 2009; larval zebrafish, McHenry et al., 2009; killifish, Schwarz et al., 2011; and another cichlid, the oscar, Astronotus ocellatus, Mogdans and Nauroth, 2011). This work showed that $A$. stuartgranti is quite sensitive to artificial flows, responding to flows with velocities as low as $\sim 1 \mathrm{~mm} \mathrm{~s}^{-1}$, which is slower than those generated by copepods and copepodids (van Duren and Videler, 2003; Catton et al., 2007), tethered krill (Yen et al., 2003), midge larvae in burrows (Morad et al., 2010), and the tethered brine shrimp used with $A$. stuartgranti in prior studies (Schwalbe et al., 2012).

It appears that $A$. stuartgranti can discriminate between different types of hydrodynamic stimuli. In the current study, fish typically responded to pulsed flows with a hover response often accompanied by a bite response (in $\sim 65 \%$ of responses). However, Schwalbe (Margot A. B. Schwalbe, The role of flow sensing by the lateral line system in prey detection in two African cichlid fishes, $\mathrm{PhD}$ thesis, 

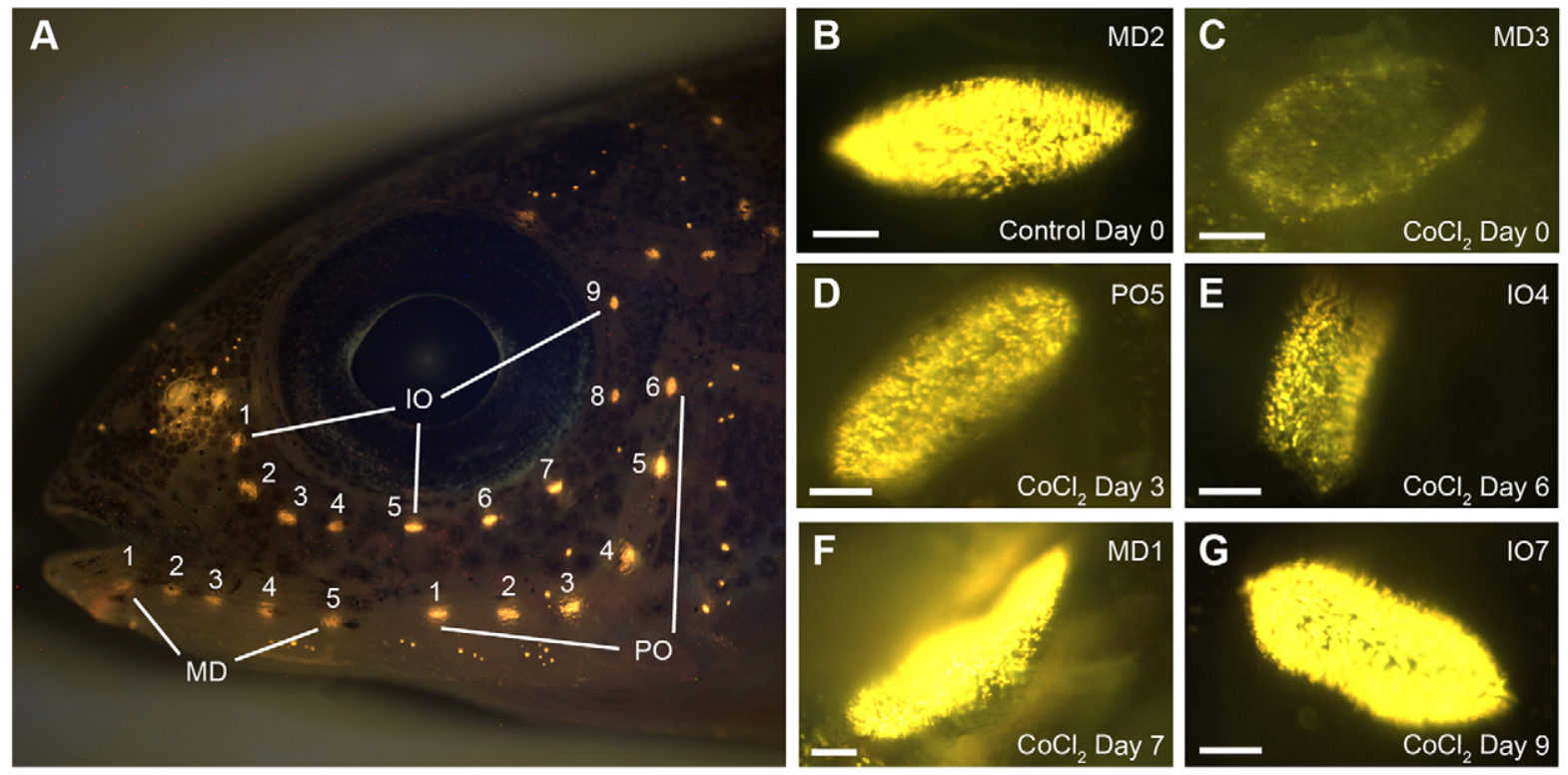

Fig. 7. Fluorescence staining of hair cells in neuromasts of $\boldsymbol{A}$. stuartgranti. (A) Lateral view of a juvenile fish (25 mm SL) showing hair cells in canal neuromasts (larger ovals) and superficial neuromasts (smaller dots) fluorescently stained with DASPEI. (B-G) Staining of hair cells in canal neuromasts using 4Di-2-ASP, demonstrating recovery from $\mathrm{CoCl}_{2}$ treatment in $A$. stuartgranti over 9 days. (B) Day 0 control; (C) day 0 post-treatment; (D) day 3 post-treatment; (E) day 6 post-treatment; (F) day 7 post-treatment; and (G) day 9 post-treatment. Scale bars, $100 \mu \mathrm{m}$. MD, mandibular canal neuromast; IO, infraorbital canal neuromast; PO, preopercular canal neuromast. Neuromast identification based on Becker (E. A. Becker, The distribution and ontogeny of neuromast receptor organs and a comparison of methods for chemical ablation of the lateral line system in two cichlid fishes, MSc thesis, University of Rhode Island, 2013).

University of Rhode Island, 2013) showed the same group of fish used in this study tended to bite less frequently (4-20\% of responses) when these pulses were dampened, even though they detected these dampened flows at similarly high frequencies (68-89\% response rate). In a similar manner, the mottled sculpin (Cottus bairdi) responded unconditionally to a 'pulsed flow' (generated by a syringe connected to a minishaker), but not to a 'laminar flow' (generated by a gravity-fed system; Janssen et al., 1990). If different prey species generate water flows that vary in hydrodynamic structure, either spatially or temporally, a predator's ability to distinguish among them could have important ecological implications for the definition of the trophic niches occupied by fishes.

The use of artificial flows allowed the contribution of the lateral line system to flow sensing in $A$. stuartgranti to be interpreted in the absence of the confounding effects of visual and olfactory input generated by live prey (Schwalbe et al., 2012; Schwalbe and Webb, 2014). The high frequency of responses to the active hole (in trials with flow; Fig. 3A) compared with responses to the other three hole types (the $\mathrm{VC} 1$ hole, in trials with flow; the inactive hole and the VC2 hole, in trials with diverted flow) clearly demonstrated the ability of fish to detect flows at all flow velocities presented. Nevertheless, some fish occasionally responded to holes other than the active hole. Care had been taken to reduce noise by allowing the pump to run during all trials (including those with diverted flow), limiting the propagation of vibrations in the experimental tank by placing the pump on a separate table, and shielding the tubing from the experimental arena in the tank. Thus, responses to inactive holes (in trials with diverted flow) were not likely triggered by variation in acoustic or hydrodynamic noise. However, the position of the active hole to the left of its paired visual control hole (VC1 in trials with flow) was consistent among all trials, so fish may have learned to pause over the left tube in the pair, regardless of whether flow was present. In addition, hovering over holes other than the active hole may just indicate failed attempts to detect flow motivated by the promise of a food reward.

Finally, this study has demonstrated that the effects of cobalt chloride treatment are reversible (but not immediately), and that the use of cobalt chloride is an effective method for experimentally determining the flow-sensing function of the lateral line system. Karlsen and Sand (1987) showed that the roach Rutilus rutilus (a cyprinid) treated with cobalt chloride $\left(0.1 \mathrm{mmol}^{-1}\right.$ for $\left.24 \mathrm{~h}\right)$ recovered some lateral line function after a few hours in a recovery tank (when stimulated by a massive water jet from a pipette) and fully recovered after 2-3 weeks (indicated by normal behavioral sensitivity to a vibrating sphere). In a previous study (Schwalbe et al., 2012), we showed that $A$. stuartgranti recovers most aspects of prey detection behavior in response to tethered brine shrimp at a point 3-4 weeks after treatment (Schwalbe et al., 2012), but behavior was not assessed between the day of treatment and 3-4 weeks after treatment. In the current study, daily behavioral sessions and periodic visualization of neuromast fluorescence posttreatment allowed a more precise description of the course of recovery. For instance, immediately after treatment (day 0), fish showed few responses to an active hole despite swimming directly over it (24\%; Fig. 5A). Fish demonstrated a much higher $(71.1 \%)$ response rate to the active hole by day 2 , and fluorescence due to staining with 4-di-2-ASP (indicating live, functional sensory hair cells) returned in superficial neuromasts by day 3 (M.A.B.S. and E. A. Becker, unpublished data; see also Emily A. Becker, The distribution and ontogeny of neuromast receptor organs and a comparison of methods for chemical ablation of the lateral line system in two cichlid fishes, MSc thesis, University of Rhode Island, 2013) and in canal neuromasts (with a larger hair cell population) by day 7 . This time frame is consistent with the course of hair cell regeneration in the lateral line system of larval zebrafish and in the ear of other vertebrates (reviewed in Brignull et al., 2009; Lush and Piotrowski, 2014; Atkinson et al., 2015). The correlation 
of loss and then recovery of both lateral line-mediated behavior and morphological indicators of hair cell functionality after treatment with cobalt chloride, and evidence that it does not adversely affect other sensory systems (Yoshii and Kurihara, 1983; Pohlmann et al., 2004; Liao, 2006), provides strong evidence that the effects of cobalt chloride (at the concentration used in this study, $0.1 \mathrm{mmol} \mathrm{l}^{-1}$ for $3 \mathrm{~h}$ ) are specific to the lateral line system and not indicative of general toxic effects (as had been suggested by Janssen, 2000).

In conclusion, $A$. stuartgranti was successfully trained to identify artificial water flows emerging from a sandy substrate, which served as proxies for the benthic invertebrate prey that they normally consume in Lake Malawi. The study demonstrated that fish can identify these ecologically relevant flows in the absence of input to other sensory modalities, and that they respond with a range of quantifiable behaviors. The role of the lateral line system in flow sensing was confirmed experimentally and correlated with a morphological indicator of hair cell function. The return of flowsensing behaviors over a period of a week after inactivation was consistent with a process of hair cell regeneration, providing a link between sensory cell function and whole-animal behavior. These results present new opportunities to study the role of flow sensing as well as feeding ecology in benthic feeding fishes.

\section{Acknowledgements \\ We thank undergraduate ocean engineering students Kevin Borsay and Adam Arrighi for designing, constructing and testing the flow presentation apparatus used in this study and Brett Bergstrom for constructing the manual switch for the peristaltic pump used in this study. We thank Dr Cheryl Wilga who provided expertise and allowed us to use her DPIV equipment, Edward Baker (Facilities Manager, RI NSF EPSCoR Marine Life Science Facility), Dr Eric Tytell for helpful feedback on the manuscript, and Emily Becker, Christopher Holland and Rebecca Scott who provided assistance with fish husbandry.}

\section{Competing interests}

The authors declare no competing or financial interests.

\section{Author contributions}

M.A.B.S. designed the fish training protocol and experiments, collected behavioral and morphological data, carried out statistical analysis, and wrote the manuscript. B.J.S. contributed to the development of the training protocol, and collection of behavioral data. J.F.W. contributed to the experimental design and to the writing of the manuscript.

\section{Funding}

This work was funded by National Science Foundation (NSF) grant IOS 08-43307 to J.F.W., a University of Rhode Island Graduate Research Fellowship to M.A.B.S., the College of the Environment and Life Sciences (University of Rhode Island), and NSF EPSCoR Cooperative Agreements EPS-0554548 and EPS-1004057.

\section{References}

Atkinson, P. J., Najarro, E. H., Sayyid, Z. N. and Cheng, A. G. (2015). Sensory hair cell development and regeneration: similarities and differences. Development 142, 1561-1571.

Bird, N. C. and Webb, J. F. (2014). Heterochrony, modularity, and the functional evolution of the mechanosensory lateral line canal system of fishes. EvoDevo 5, 21.

Bleckmann, H. (1980). Reaction time and stimulus frequency in prey localization in the surface-feeding fish Aplocheilus lineatus. J. Comp. Physiol. A 140, 163-172.

Bleckmann, H., Waldner, I. and Schwartz, E. (1981). Frequency discrimination of the surface-feeding fish Aplocheilus lineatus - a prerequisite for prey localization? J. Comp. Physiol. A 143, 485-490.

Brignull, H. R., Raible, D. W. and Stone, J. S. (2009). Feathers and fins: nonmammalian models for hair cell regeneration. Brain Res. 1277, 12-23.

Catton, K. B., Webster, D. R., Brown, J. and Yen, J. (2007). Quantitative analysis of tethered and free-swimming copepodid flow fields. J. Exp. Biol. 210, 299-310.

Claas, B., Münz, H. and Görner, P. (1993). Reaction to surface waves by Xenopus laevis Daudin. Are sensory systems other than the lateral line involved? J. Comp. Physiol. A 172, 759-765.

Coombs, S. and Janssen, J. (1990). Behavioral and neurophysiological assessment of lateral line sensitivity in the mottled sculpin, Cottus bairdi. J. Comp. Physiol. A 167, 557-567.
Coombs, S. and Montgomery, J. (2014). The role of flow and the lateral line in the multisensory guidance of orienting behavior. In Flow Sensing in Air and Water (ed. H. Bleckmann, J. Mogdans and S. L. Coombs), pp. 65-101. Berlin: SpringerVerlag.

Coombs, S., Janssen, J. and Webb, J. F. (1988). Diversity of lateral line systems: evolutionary and functional considerations. In Sensory Biology of Aquatic Animals (ed. J. Atema, R. R. Fay, A. N. Popper and W. N. Tavolga), pp. 553-593. New York: Springer-Verlag.

Coombs, S., Braun, C. B. and Donovan, B. (2001). The orienting response of Lake Michigan mottled sculpin is mediated by canal neuromasts. J. Exp. Biol. 204, 337-348.

Denton, E. J. and Gray, J. A. B. (1988). Mechanical factors in the excitation of the lateral lines of fish. In Sensory Biology of Aquatic Animals (ed. J. Atema, R. R. Fay, A. N. Popper and W. N. Tavolga), pp. 595-617. New York: Springer-Verlag.

Denton, E. J. and Gray, J. A. B. (1989). Some observations on the forces acting on neuromasts in fish lateral line canals. In The Mechanosensory Lateral Line: Neurobiology and Evolution (ed. S. Coombs, P. Gorner and H. Münz), pp. 229-246. New York: Springer-Verlag.

Hoekstra, D. and Janssen, J. (1985). Non-visual feeding behavior of the mottled sculpin, Cottus bairdi, in Lake Michigan. Env. Biol. Fish. 12, 111-117.

Hoekstra, D. and Janssen, J. (1986). Lateral line receptivity in the mottled sculpin (Cottus bairdi). Copeia 1986, 91-96.

Janssen, J. (1997). Comparison of response distance to prey via the lateral line in the ruffe and yellow perch. J. Fish Biol. 51, 921-930.

Janssen, J. (2000). Toxicity of $\mathrm{Co}^{2+}$ : implications for lateral line studies. J. Comp. Physiol. A Sens. Neural. Behav. Physiol. 186, 957-960.

Janssen, J., Coombs, S. and Pride, S. (1990). Feeding and orientation of mottled sculpin, Cottus bairdi, to water jets. Environ. Biol. Fish. 29, 43-50.

Jordan, L. K., Kajiura, S. M. and Gordon, M. S. (2009). Functional consequences of structural differences in stingray sensory systems. Part I: mechanosensory lateral line canals. J. Exp. Biol. 212, 3037-3043.

Karlsen, H. E. and Sand, O. (1987). Selective and reversible blocking of the lateral line in freshwater fish. J. Exp. Biol. 133, 249-262.

Konings, A. (1990). Koning's Book of Cichlids and Other Fishes of Lake Malawi. Neptune City, NJ: TFH Publications Inc.

Konings, A. (2007). Malawi Cichlids in Their Natural Habitat, 4th edn. El Paso, TX Cichlid Press.

Liao, J. C. (2006). The role of the lateral line and vision on body kinematics and hydrodynamic preference of rainbow trout in turbulent flow. J. Exp. Biol. 209, 4077-4090.

Lush, M. E. and Piotrowski, T. (2014). Sensory hair cell regeneration in the zebrafish lateral line. Dev. Dyn. 243, 1187-1202.

McHenry, M. J., Feitl, K. E., Strother, J. A. and Van Trump, W. J. (2009) Larval zebrafish rapidly sense the water flow of a predator's strike. Biol. Lett. $\mathbf{5}$, 477-479.

Mogdans, J. and Nauroth, I. E. (2011). The oscar, Astronotus ocellatus, detects and discriminates dipole stimuli with the lateral line system. J. Comp. Physiol. A 197, 959-968.

Montgomery, J. and Skipworth, E. (1997). Detection of weak water jets by the short-tailed stingray Dasyatis brevicaudata (Pisces: Dasyatidae). Copeia 1997, 881-883.

Montgomery, J. C., Bleckmann, H. and Coombs, S. (2014). Sensory ecology and neuroethology of the lateral line. In The Lateral Line System (ed. S. Coombs and H. Bleckmann), pp. 121-150. New York: Springer-Verlag.

Morad, M. R., Khalili, A., Roskosch, A. and Lewandowski, J. (2010) Quantification of pumping rate of Chironomus plumosus larvae in natural burrows. Aquat. Ecol. 44, 143-153.

Nauroth, I. E. and Mogdans, J. (2009). Goldfish and Oscars have comparable responsiveness to dipole stimuli. Naturwissenschaften 96, 1401-1409.

Palmer, L. M., Deffenbaugh, M. and Mensinger, A. F. (2005). Sensitivity of the anterior lateral line to natural stimuli in the oyster toadfish, Opsanus tau (Linnaeus). J. Exp. Biol. 208, 3441-3450.

Pohlmann, K., Atema, J. and Breithaupt, T. (2004). The importance of the latera line in nocturnal predation of piscivorous catfish. J. Exp. Biol. 207, 2971-2978.

Schwalbe, M. A. B. and Webb, J. F. (2014). Sensory basis for detection of benthic prey in two Lake Malawi cichlids. Zoology 117, 112-121.

Schwalbe, M. A. B. and Webb, J. F. (2015). The effect of light intensity on prey detection behavior in two Lake Malawi cichlids, Aulonocara stuartgranti and Tramitichromis sp. J. Comp. Physiol. A. 201, 341-356.

Schwalbe, M. A. B., Bassett, D. K. and Webb, J. F. (2012). Feeding in the dark: lateral-line-mediated prey detection in the peacock cichlid Aulonocara stuartgranti. J. Exp. Biol. 215, 2060-2071.

Schwarz, J. S., Reichenbach, T. and Hudspeth, A. J. (2011). A hydrodynamic sensory antenna used by killifish for nocturnal hunting. J. Exp. Biol. 214, 1857-1866.

van Duren, L. A. and Videler, J. J. (2003). Escape from viscosity: the kinematics and hydrodynamics of copepod foraging and escape swimming. J. Exp. Biol. 206 269-279. 
Webb, J. F. (2014). Morphological diversity, development, and evolution of the mechanosensory lateral line system. In The Lateral Line System (ed. S. Coombs and H. Bleckmann), pp. 17-72. New York: Springer-Verlag.

Yen, J., Brown, J. and Webster, D. R. (2003). Analysis of the flow field of the krill, Euphausia pacifica. Mar. Fresh. Behav. Physiol. 36, 307-319.
Yoshii, K. and Kurihara, K. (1983). Role of cations in olfactory reception. Brain Res. 274, 239-248.

Yoshizawa, M., Gorički, Š., Soares, D. and Jeffery, W. R. (2010). Evolution of a behavioral shift mediated by superficial neuromasts helps cavefish find food in darkness. Curr. Biol. 20, 1631-1636. 